Running title: Adaptations to cognitive problems in breast cancer survivors

**PRE-PRINT: This is the accepted version of the following article: Green HJ, Mihuta ME, Ownsworth T, Dhillon HM, Tefay M, Sanmugarajah J, Tuffaha HW, Ng S-K, Shum DHK. Adaptations to cognitive problems reported by breast cancer survivors seeking cognitive rehabilitation: A qualitative study. Psycho-Oncology. 2019; 1-7, which has been published in final form at https://doi.org/10.1002/pon.5189**

Adaptations to cognitive problems reported by breast cancer survivors seeking cognitive rehabilitation: A qualitative study

Heather J. Green ${ }^{1}$, Mary E. Mihuta ${ }^{1}$, Tamara Ownsworth ${ }^{2}$, Haryana M. Dhillon ${ }^{3}$, Merilyn Tefay $^{4}$, Jasotha Sanmugarajah ${ }^{5}$, Haitham W. Tuffaha ${ }^{6}$, Shu-Kay $\mathrm{Ng}^{6}$, \& David H. K. Shum ${ }^{2,7}$

${ }^{1}$ School of Applied Psychology and Menzies Health Institute Queensland, Griffith University, Gold Coast, QLD, Australia

${ }^{2}$ School of Applied Psychology and Menzies Health Institute Queensland, Griffith University, Brisbane, QLD, Australia

${ }^{3}$ Centre for Medical Psychology and Evidence-Based Decision-Making, University of Sydney, Sydney, NSW, Australia

${ }^{4}$ Mater Health Services, Brisbane, QLD, Australia

${ }^{5}$ Department of Medical Oncology, Gold Coast University Hospital, Gold Coast, QLD, Australia

${ }^{6}$ School of Medicine and Menzies Health Institute Queensland, Griffith University, Brisbane, QLD, Australia

${ }^{7}$ Department of Rehabilitation Sciences, The Hong Kong Polytechnic University, Hong Kong, People's Republic of China

Correspondence: Dr Heather Green, School of Applied Psychology, Griffith University, Gold Coast, QLD, Australia. Email: h.green@griffith.edu.au

Funding: Funders were Mater Foundation (Project 1337); School of Applied Psychology, Griffith University; and Department of Science, Information Technology and Innovation, Queensland Government (WAF-7888469-32). During data collection, Mary Mihuta was supported by a joint PhD scholarship provided by Cancer Council Queensland and Griffith University.

Acknowledgements: This research was supported by Breast Cancer Network Australia's Review \& Survey Group, a national, online group of Australian women living with breast cancer who are interested in receiving invitations to participate in research. We acknowledge the women involved in the Review and Survey Group who participated in this project.

Conflicts: None 


\begin{abstract}
Objective. Qualitative studies have elucidated cancer survivors' experiences of cognitive changes associated with cancer and cancer treatment. This study specifically explored experiences of women treated for breast cancer who were seeking cognitive rehabilitation. The objective was to characterise the frequency and nature of cognitive changes and adaptations to cognitive change reported by these participants, to better understand treatment needs of this group.
\end{abstract}

Method. Australian women who had completed primary treatments for breast cancer (surgery, chemotherapy, and/or radiotherapy) and volunteered to participate in one of two cognitive rehabilitation intervention studies were interviewed via telephone.

Interview responses regarding cognitive changes and adaptations to cognitive change were transcribed by the interviewers, then coded and analysed by two researchers using content analysis.

Results. Among the 95 participants (age $M=54.3$ years, $S D=9.6$ ), the most commonly reported cognitive change was memory (79\% of participants) and $61 \%$ reported more than one type of cognitive change. Adaptations to change were reported by $87 \%$ of participants, with written or electronic cues the most common (51\%). Most often, participants reported using a single type of adaptation (48\%) with only $39 \%$ reporting multiple types of adaptations.

Conclusions. Women treated for breast cancer who were seeking cognitive rehabilitation most commonly reported memory changes which were mainly managed through a single type of adaptation. These results suggest that there is considerable scope for increasing the range of cognitive adaptations, to improve the quality of life of cancer survivors who experience adverse cognitive changes.

Keywords: Cancer; oncology; cognitive impairment; coping strategies; qualitative; breast cancer; chemotherapy; cognitive rehabilitation

Data Availability Statement: The data that support the findings of this study are available on request from the corresponding author. The data are not publicly available due to privacy or ethical restrictions. 


\section{Cognitive problems and adaptations reported by breast cancer survivors seeking cognitive rehabilitation: A qualitative study}

Cancer-related cognitive impairment (CRCI) is experienced in conjunction with cancer and/or cancer treatments by approximately $30 \%$ of patients with noncentral nervous system malignancy before beginning treatment; as many as $75 \%$ during treatment; and up to $35 \%$ after treatment completion [1]. Pragmatic recommendations regarding treatments have been made [2], but there is currently no broadly adopted evidence-based treatment and a paucity of research addressing translation of treatments into practice [3]. Although neuropsychological assessments might be viewed as ideal for detecting and monitoring CRCI, this is not practically feasible on a large scale [2] and thus self-report is likely to remain the main format for assessing subjective cognitive concerns. Along with validated measures of selfreported CRCI [4], qualitative information provided by patients on their everyday experiences continues to be an important means by which CRCI concerns come to light.

A key qualitative study of 74 breast cancer survivors who experienced symptoms persisting for more than 12 months after completing adjuvant chemotherapy and/or radiotherapy found cognitive impairment was one of the most frequently reported ongoing symptoms, mentioned by $70 \%$ of participants [5]. These women reported that cognitive changes were "frustrating" and "upsetting". These changes included problems with recall, word-finding, speed of processing, concentrating, and complex tasks such as reading, driving, and paying bills. Reported impacts on work included reduced speed and efficiency, decreased competitiveness for selection or promotion, problems in studying to re-enter the workforce, changing to a less cognitively demanding occupation with reduced salary, and retiring early [5]. Coping strategies included use of devices such as journals, calendars, reminder notes, and routines; mentally challenging activities such as mathematics problems or formal study; and lifestyle changes such as reducing work and social activities or alerting others to their cognitive problems [5].

An interview study specific to memory and concentration among 93 women who received chemotherapy for breast cancer and 49 whose treatment did not involve chemotherapy found higher rates of reported changes in memory and concentration among women who received chemotherapy than those who did not [6]. Reports of 
problems in memory and concentration decreased in both groups by 12 months postchemotherapy or the equivalent time point in the non-chemotherapy group [6].

Systematic reviews or meta-synthesis of qualitative studies [7-9] and other individual qualitative studies with smaller numbers of breast cancer survivors [10-20] have reported similar findings. For example, semi-structured interviews with 18 breast cancer survivors, followed by a focus group, revealed that participants reported problems with short-term memory, word finding, concentration, and poorer performance in work, study, driving, and financial management [14]. Coping strategies included using reminders such as written and/or electronic prompts, seeking assistance and support from others, exercise, rest, and mental stimulation [14].

Most qualitative studies to date have included relatively small samples of participants (i.e., $n<20$ ), and some have focused on specific cognitive problems or specific contexts such as work performance. In the present study, 95 breast cancer survivors who reported cognitive changes and were seeking cognitive rehabilitation as part of a clinical trial [3,21] were interviewed about the nature of cognitive changes and adaptations they made in response to cognitive changes. The aim was to describe, for the first time, the cognitive concerns of cancer survivors who are seeking treatment to remedy these problems as well as strategies they had employed before rehabilitation treatment. It was expected that this research would provide insight into treatment needs.

\section{Methods}

\section{Design}

Planning, conduct, and reporting of this study followed the 32-item checklist in Consolidated Criteria for Reporting Qualitative Research (COREQ) guidelines [22]. Data were collected using a semi-structured telephone interview. Directed content analysis was used to extract themes and frequency of different cognitive issues and adaptations [23]. This analysis uses existing theory or research findings to guide initial selection and operationalisation of codes and permits additional codes to be derived from the data inductively [23]. Directed content analysis was chosen due to the context of relevant prior theory and research and suitability to identify frequency within qualitative data.

\section{Recruitment}

Participants were recruited for one of two intervention trials of cognitive rehabilitation, following hospital and university ethics approval (Study 1 approvals 
HREC/15/MHS/10 and PSY/88/15/HREC; Study 2 PSY/F4/14/HREC): (1) a single arm trial of the Responding to Cognitive Concerns ( $\mathrm{ReCog}$ ) small group intervention ( $n=25$ recruited at Mater Cancer Care Centre, Brisbane, Australia between June 2015 and October 2016); and (2) a randomised waitlist-controlled trial of the individually completed web-based format, $e \operatorname{ReCog}(n=70$ recruited via Breast Cancer Network Australia and Griffith University email lists between April and June 2016).

Participants who had completed primary treatments for breast cancer (surgery, radiotherapy, and/or chemotherapy) and who completed screening and baseline assessments were eligible for the present study. Further details of eligibility and recruitment are available in the published findings of the intervention studies[3,21].

\section{Procedures}

Participants recruited for the in-person $(\mathrm{ReCog})$ trial were approached via mail or clinical staff contact. Participants from the web-based $(e \operatorname{ReCog})$ trial were approached via email. After providing informed consent, participants undertook a semi-structured telephone interview of approximately 20 minutes duration which both provided the data for the present study and served as a screening process to check eligibility for the subsequent intervention trials. Interviews were conducted by a female clinical psychologist/academic (HG) and a female provisionally registered psychologist/PhD candidate (MM) who each had clinical and research experience in psycho-oncology regarding cognitive difficulties. Participants had the opportunity to review written information sheets and to ask questions before proceeding with the interview but otherwise had no prior knowledge about the interviewers.

Participants completed telephone interviews at locations convenient to them (e.g., home, work). Interviews involved the following sections: (1) rapport establishment and informed consent, (2) screening with the cognitive functioning subscale from the European Organisation for Research and Treatment of Cancer Core Quality of Life Questionnaire (EORTC-QLQ-C30)[24], (3) Demographic information, (4) Medical information, (5) Cognitive changes and adaptations, and (6) Final questions from participant and closure. The focus of this analysis was Section 5, where participants were asked "Do you think that you have experienced any cognitive difficulties that were directly related to your cancer or your cancer treatment?" If they said yes, they were asked for the most severe example and whether changes were still current. They were also asked "If you experienced changes, what adaptations did you have to make in areas of your life?" If elaboration or clarification was needed, 
interviewers used prompts such as "Tell me more about that" or "What did you do differently because of these changes, if anything?" Interview questions are detailed in the supplementary appendix. Interviewers typed responses into MS Word during the interviews.

\section{Data Analyses}

Demographic and medical data were transcribed into SPSS for analysis, and interview data for Section 5 were transcribed into MS Excel. Data were independently coded by the two interviewers, $\mathrm{HG}$ and $\mathrm{MM}$, using a codebook that was drafted by HG after discussion with DS, reviewed by TO and HD, and refined iteratively by both coders. The first step in directed content analysis [23] was to segment participants' responses into separate concepts (e.g., "working part time" was segmented as a separate adaptation from "picked up yoga and meditation again, doing regularly"). Next, cognitive problems were coded using classifications of cognitive functions from a core neuropsychology text [25] (e.g., "struggled and am still struggling to get my concentration back" was coded in attention functions; "problems thinking of words" was coded in verbal functions). A priori cognitive themes or types of change followed Lezak et al. [25] except that executive functions incorporated concept formation and reasoning [26]. As permitted in directed content analysis, additional themes were derived inductively from the data, including all adaptation themes. Then, reliability of coding was checked, showing that coders agreed on the presence or absence of each cognitive theme in $97 \%$ of classifications and the presence or absence of each adaptation theme in $97 \%$ of classifications. Last, disagreements between coders were resolved through consensus. The codebook is available from the corresponding author.

\section{Results}

Ninety-five female participants completed the telephone interview. Participants' mean age was 54.3 years ( $S D=9.6$, range: $27-72)$. They had completed an average of 14.5 years of education ( $S D=2.4$, range: $9-19)$ and on average finished primary treatment 40.9 months previously $(S D=40.1$, range: $2-171)$. All participants had surgery for treatment, $86 \%$ had chemotherapy, $73 \%$ had radiotherapy, $76 \%$ received hormone therapy, and $22 \%$ received targeted therapy such as trastuzumab.

We found that participants reported up to 4 types of cognitive change and up to 4 types of adaptation. Table 1 shows the percentage of participants with responses 
classified into each theme for types of cognitive change and adaptation to cognitive changes.

\section{Cognitive Change Themes}

Types of Cognitive Change. The most common cognitive change reported was memory (79\%), e.g., "very forgetful - could not remember the next day what I had done" or "forgetting appointments". The next most frequent was attention (39\%), e.g., "my focus is not so good" or "I couldn't seem to read, focus or concentrate. I would pick up a book and usually I could keep reading, but I would read one or two paragraphs and could not concentrate or remember what I had read". This was followed by the verbal functions theme (28\%), e.g., "my speech was very slow" or “it's like a nominal aphasia, I can't think of the names of things". Executive function changes were reported by $14 \%$, e.g., "I have to concentrate really hard to get my brain to think on multiple levels and do multiple tasks" or "I'm doing one thing and move on to another without finishing, which is something I never did before. I have to constantly drag myself to finish something". Six participants reported worse processing speed, i.e., "needing more time/thinking to process instructions" and "not as fast as I used to be". One participant reported changed orientation, i.e., "I've been driving on a route I've driven on many times, and have the feeling I've never been there before, and have a panic. So something where the brain just seems to slip a little, not severe, but enough to make an impact". One participant reported a change in perceptual cognition, i.e., "I have some perception problems, judging distances and speeds (driving)". Some participants (12\%) reported global or non-specific cognitive decline, e.g., "I think it's just a diminished ability to think" or "foggy brain".

\section{INSERT TABLE 1 ABOUT HERE}

Other Change Types. When asked about cognitive changes, $18 \%$ of participants mentioned changes in emotions (18\%), e.g., "I find it [the cognitive changes] very distressing". Fatigue was mentioned by $9 \%$ of participants, e.g., "I still get really tired, incredibly tired". Four participants reported problems with sleep, e.g., "I can't sleep properly, I feel like my mind is going everywhere, I have to sit back up to try to get my memory back." Six participants reported non-cognitive changes other than in emotions, fatigue, and sleep, e.g., "I have dizziness" and "on Tamoxifen - had extensive swelling (feet swollen, eyes sore)". One of the participants categorised as 
reporting "other" change described unusual thoughts: "Yes certainly the second time with chemotherapy. I have terrible sort of delusions. For example, I thought I had lost my body, only my head was there, and it took me the whole day standing in front of the mirror to convince myself that I have my body. I was pinching myself, etc. I have also had trouble remembering peoples' faces, but couldn't. [It] went on a little after the chemotherapy."

Contexts and Impacts. A frequent theme when participants were asked about cognitive change (mentioned by $45 \%$ of participants) was their ideas about what caused their cognitive problems, including ambiguity about causes, e.g., "seems to have gotten much worse since the chemo and Aromasin" or "I do notice more problems with concentration and recall, I'm not sure if it's my age, or genetics, or my treatment." Occupational impacts on work or study were mentioned by $17 \%$ and $2 \%$ of participants respectively, e.g., "I already had a complex job before, but since coming back in February I have to concentrate really hard to get my brain to think on multiple levels and do multiple tasks. Since February, it's been very hard. I don't feel like I am back to before" or "I found last year that I actually failed two courses at uni, I wasn't able to remember the information." Social impacts regarding family and friends were raised by $8 \%$ of participants, e.g., "Maths freaks me out, I have an 8 year old daughter, and I can't help her with her maths because I find it very distressing" or "Losing friends - can't go out at night (very fatiguing - have to sleep during the day first)".

\section{Adaptation Themes}

Of the adaptations reported by participants (Table 1), using written or electronic cues were the most common (51\%), e.g., "Making many lists, putting information in the phone calendar (e.g., medication reminders)" or "write things down more, much more diligent in writing things down rather than relying on memory". A group of adaptations we labelled "task and environmental accommodations" was the next most common (32\%), involving processes such as reducing distractions, taking more time, taking breaks from cognitive tasks before coming back, and reduced multi-tasking, e.g., "try to focus on one thing at a time" or "at work, I have to break down my tasks and do parts, do something else, then come back to it because I just can't maintain my attention". Changes in lifestyle or health behaviours were reported by $21 \%$, e.g., "Only drive on a good day" or "I exercise more, lost some weight". Adaptations of an interpersonal nature (15\%) included "get 
my husband to write things in his diary and remind me" and "avoid talking to people". Adaptations in work due to cognitive changes were reported by $14 \%$ of participants and most often involved reducing work hours, e.g., "We needed to employ someone else in the business" or "now working only one day a week rather than four". Other work adaptations included changing roles, leaving the workforce, and working longer hours to get tasks completed (e.g., "lots of hours of unpaid overtime"). Acceptance of change or altering self-expectations was an adaptation mentioned by six participants, e.g., "I use a lot of patience and tolerance" or "I just keep going on, and if I forget something, I just do". Undertaking cognitively stimulating activities was mentioned by three participants, e.g., "my friend has me on the internet now and there are little games I can play, which I have been doing over the past few weeks" or "I took up cryptic crosswords, puzzle solving, made sure I did at least 5 minutes per day”. One participant mentioned receiving professional help for cognitive problems, "I participated in a sleep study, after chemo I was tired all the time, it was at a cancer survivor centre. It taught cognitive strategies et cetera".

In terms of the frequency and nature of cognitive changes (Figure 1), all participants except one reported at least one type of cognitive change, and $61 \%$ reported more than one type of cognitive change. The frequency distribution was different for adaptations, where $13 \%$ reported no adaptations, $48 \%$ reported only one type of adaptation, and 39\% of participants reported multiple types of adaptations.

\section{INSERT FIGURE 1 ABOUT HERE}

\section{Conclusions}

Overall, this study identified that women treated for breast cancer who were seeking cognitive rehabilitation perceived multiple cognitive changes, yet individuals tended to rely on only one type of adaptation. Although memory was the most frequent cognitive change theme, multiple themes were identified and these often cooccurred. The types of problems reported were consistent with those found in other qualitative studies [5,7-10,12-20]. The co-occurring cognitive problems suggest screening, assessment, and management approaches for cancer survivors should incorporate domains beyond memory and attention. Overlap between cognitive change and symptoms such as distress and fatigue was reported by a number of 
women and is consistent with evidence that cognitive impairment can have bidirectional relationships with both distress and fatigue [27].

Whilst clinical researchers have previously suggested that most cancer survivors concerned about cognitive function use compensatory strategies [2], this is the first study to describe what proportion of participants reports using such strategies. Indeed, $87 \%$ of participants reported making adaptations, of types consistent with previous research $[5,11,14]$. However, it is noteworthy that $13 \%$ of participants had not made any adaptations, which may account for their interest in participating in cognitive rehabilitation research. Relatedly, $48 \%$ of participants reported only one type of adaptation, so whilst they might use several types of cueing or several types of lifestyle change, they did not use adaptations from other categories. This indicates that there were many participants with potential to benefit from a broader range of adaptations and/or receiving assistance in implementing such adaptations. Participants in the present study all had the opportunity to subsequently take part in either face-toface $\mathrm{ReCog}$ or web-based $e \mathrm{ReCog}$, an education and skills training program for cancer survivors that facilitates use of a variety of compensatory and/or enhancement strategies shown to improve self-reported cognitive function [3,21]. Results of these intervention studies have been published [3,21].

\section{Study Limitations}

In Section 2 of the telephone interview, participants were verbally administered the cognitive functioning subscale from EORTC-QLQ-C30 asking specifically about memory and concentration. There were then intervening questions about demographic and medical history, but it is possible that the standard items about memory and concentration influenced the examples participants gave of perceived cognitive problems. For example, the standardised concentration item specifically mentions reading, which might have made difficulties with reading more salient and thus readily retrievable. Another limitation is that, due to resource constraints associated with the sample size $(N=95)$, it was not possible to record the interviews as is typical in qualitative research. Both interviewers aimed to record participants' responses as fully as possible; however, it is possible that some interview responses were not captured. Recording and verbatim transcription of the 95 interviews would have improved methodological rigour. The direct prompt about cognitive problems was for "the most severe example"; many participants gave multiple examples but it is possible that this wording limited the range of responses 
given by participants or extent of issues experienced. There was also possible selection bias given that this convenience sample involved participants who were all seeking support in the context of a cognitive rehabilitation intervention trial and most were highly educated. Nonetheless, the large sample size and recruitment through both a major hospital and consumer network is likely to enhance the broader applicability of the findings.

\section{Clinical Implications}

These results demonstrated that most women relied on one main type of adaptation to cope with multiple cognitive changes. Written and electronic cues were the main type of adaptation, suggesting participants were not aware of a broader range of potentially helpful ways to adapt to or compensate for CRCI. These results suggest that information for cancer survivors and interventions for CRCI should incorporate a range of adaptations[28]. 


\section{References}

1. Wefel JS, Kesler SR, Noll KR, Schagen SB. Clinical characteristics, pathophysiology, and management of noncentral nervous system cancer-related cognitive impairment in adults. CA Cancer J Clin 2015;65:123-138, doi:10.3322/caac.21258.

2. Vardy JL, Bray VJ, Dhillon HM. Cancer-induced cognitive impairment: practical solutions to reduce and manage the challenge. Future Oncol 2017;13:767-771, doi:10.2217/fon-2017-0027.

3. Green HJ, Tefay M, Mihuta ME. Feasibility of small group cognitive rehabilitation in a clinical cancer setting. Psycho-Oncol 2018;27:1341-1343, doi:10.1002/pon.4600. 4. Bray VJ, Dhillon HM, Vardy JL. Systematic review of self-reported cognitive function in cancer patients following chemotherapy treatment. J Cancer Surviv 2018, doi:10.1007/s11764-018-0692-x.

5. Boykoff N, Moieni M, Subramanian S. Confronting chemobrain: an in-depth look at survivors' reports of impact on work, social networks, and health care response. $J$ Cancer Surviv 2009;3:223-232, doi:10.1007/s11764-009-0098-x.

6. Shilling V, Jenkins V. Self-reported cognitive problems in women receiving adjuvant therapy for breast cancer. Eur J Oncol Nurs 2007;11:6-15, doi:10.1016/j.ejon.2006.02.005.

7. Myers JS. Cancer- and chemotherapy-related cognitive changes: The patient experience. Semin Oncol Nurs 2013;29:300-307, doi:10.1016/j.soncn.2013.08.010. 8. Duijts SF, van Egmond MP, Spelten E, van Muijen P, Anema JR, van der Beek AJ. Physical and psychosocial problems in cancer survivors beyond return to work: A systematic review. Psycho-Oncol 2014;23:481-492, doi:10.1002/pon.3467. 9. Selamat MH, Loh SY, Mackenzie L, Vardy J. Chemobrain experienced by breast cancer survivors: A meta-ethnography study investigating research and care implications. PLoS One 2014;9, doi:10.1371/journal.pone.0108002.

10. Munir F. Women's perceptions of chemotherapy-induced cognitive side affects on work ability: A focus group study. Journal of Clinical Nursing 2010;19:1362-1370, doi:10.1111/j.1365-2702.2009.03006.x.

11. Henderson FM, Cross AJ, Baraniak AR. 'A new normal with chemobrain':

Experiences of the impact of chemotherapy-related cognitive deficits in long-term breast cancer survivors. Health Psychology Open 2019;6:1-10, doi:10.1177/2055102919832234.

12. Bolton G, Isaacs A. Women's experiences of cancer-related cognitive impairment, its impact on daily life and care received for it following treatment for breast cancer. Psychol Health Med 2018;23:1261-1274, doi:10.1080/13548506.2018.1500023. 13. Munir FP, Kalawsky KB, Lawrence CM, Yarker JP, Haslam CP, Ahmed SMD. Cognitive intervention for breast cancer patients undergoing adjuvant chemotherapy: A needs analysis. Cancer Nurs 2011;34:385-392, doi:10.1097/NCC.0b013e31820254f3.

14. Myers JS. Chemotherapy-related cognitive impairment: the breast cancer experience. Oncol Nurs Forum 2012;39.

15. Rust C, Davis C. Chemobrain in underserved African American breast cancer survivors. Clinical Journal of Oncology Nursing 2013;17:E29-34, doi:10.1188/13.CJON.E29-E34. 
16. Raque-Bogdan TL, Hoffman MA, Ginter AC, Piontkowski S, Schexnayder K, White R. The work life and career development of young breast cancer survivors. Journal of Counseling Psychology 2015;62:655-669.

17. Player L, Mackenzie L, Willis K, Loh SY. Women's experiences of cognitive changes or 'chemobrain' following treatment for breast cancer: A role for occupational therapy? Australian Occupational Therapy Journal 2014;61:230-240, doi:10.1111/1440-1630.12113.

18. Sandberg JC, Strom C, Arcury TA. Strategies used by breast cancer survivors to address work-related limitations during and after treatment. Women's Health Issues 2014;24:e197-e204, doi:10.1016/j.whi.2013.12.007.

19. Kanaskie ML, Loeb SJ. The experience of cognitive change in women with breast cancer following chemotherapy. J Cancer Surviv 2015;9:375-387, doi:10.1007/s11764-014-0387-x.

20. Von Ah D, Habermann B, Carpenter JS, Schneider BL. Impact of perceived cognitive impairment in breast cancer survivors. Eur J Oncol Nurs 2013;17:236-241, doi:10.1016/j.ejon.2012.06.002.

21. Mihuta ME, Green HJ, Shum DHK. Web-based cognitive rehabilitation for survivors of adult cancer: A randomised controlled trial. Psycho-Oncol 2018;27:11721179, doi:10.1002/pon.4615.

22. Tong A, Sainsbury P, Craig J. Consolidated criteria for reporting qualitative research (COREQ): a 32-item checklist for interviews and focus groups. International Journal for Quality in Health Care 2007;19:349-357, doi:10.1093/intqhe/mzm042. 23. Hsieh H-F, Shannon SE. Three approaches to qualitative content analysis. Qual Health Res 2005;15:1277-1288, doi:10.1177/1049732305276687.

24. Aaronson NK, Ahmedzai S, Bergman B, Bullinger M, Cull A, Duez NJ, et al. The European Organization for Research and Treatment of Cancer QLQ-C30: A qualityof-life instrument for use in international clinical trials in oncology. $J$ Natl Cancer Inst 1993;85:365-376, doi:10.1093/jnci/85.5.365.

25. Lezak MD, Howieson DB, Bigler ED, Tranel D. Neuropsychological assessment (5th ed.). Oxford University Press: New York, 2012.

26. Jurado MB, Rosselli M. The elusive nature of executive functions: A review of our current understanding. Neuropsychol Rev 2007;17:213-233, doi:10.1007/s11065007-9040-z.

27. Green HJ, Pakenham KI, Gardiner RA. Cognitive deficits associated with cancer: A model of subjective and objective outcomes. Psychol Health Med 2005;10:145160, doi:10.1080/13548500500093308.

28. Dhillon H, Green H, Hutchinson A, Marston C, Vardy J, Nekkalapudi N, et al. (2018). Understanding changes in thinking and memory: Information for people affected by cancer. CAN6580 Retrieved 19 July, 2018, from https://www.cancer.org.au/content/about_cancer/treatment/CAN6580\%20Understandi ng\%20Changes\%20in\%20Thinking\%20and\%20Memory\%202018.pdf 
Table 1 Percentage of participants whose responses were classified into each theme for cognitive change types and adaptations

\begin{tabular}{lrlr}
\hline Cognitive change & $\%$ & Adaptation $^{\mathrm{b}}$ & $\%$ \\
\hline Memory $^{\mathrm{a}}$ & 79 & Written/cueing & 51 \\
Attention $^{\mathrm{a}}$ & 39 & Task and environmental accommodations & 32 \\
Verbal functions $^{\mathrm{a}}$ & 28 & Lifestyle/health & 21 \\
Executive functions $^{\mathrm{a}}$ & 14 & Interpersonal & 15 \\
Processing speed $^{\mathrm{a}}$ & 6 & Work & 14 \\
Orientation $^{\mathrm{a}}$ & 1 & Acceptance & 6 \\
Perception $^{\mathrm{a}}$ & 1 & Stimulation & 3 \\
Construction $^{\mathrm{a}}$ & 0 & Professional help & 1 \\
Motor performance $^{\mathrm{a}}$ & 0 & Other adaptation & 0 \\
Other cognitive change & 12 &
\end{tabular}

aThemes from Lezak et al.[25] with executive functions incorporating concept formation/reasoning[26]. All other themes were derived from the data.

${ }^{\mathrm{b}} \mathrm{See}$ Results section for examples of each adaptation theme. 
Adaptations to cognitive problems in breast cancer survivors 14

\section{Figure Caption.}

Frequencies of numbers of (a) cognitive change themes and (b) adaptation themes, expressed as a percentage of participants 
(a)

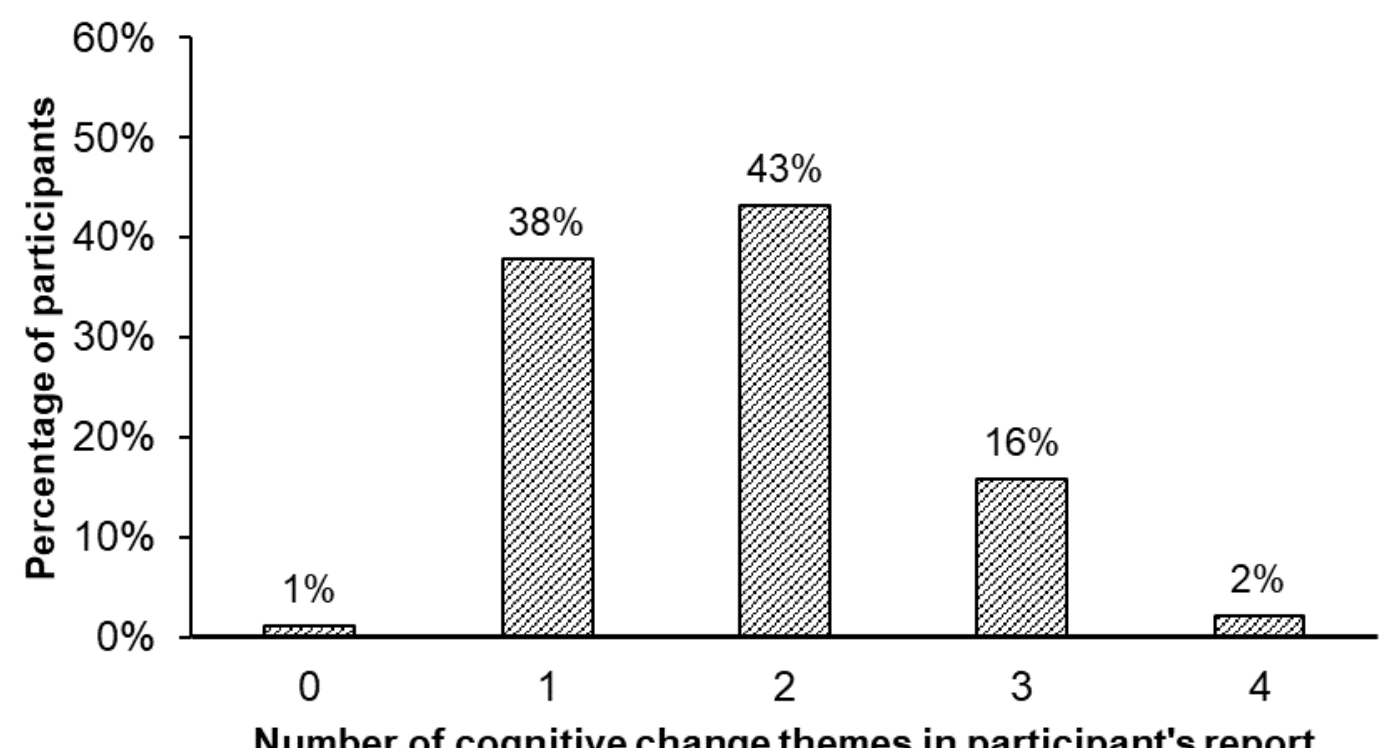

Number of cognitive change themes in participant's report

(b)

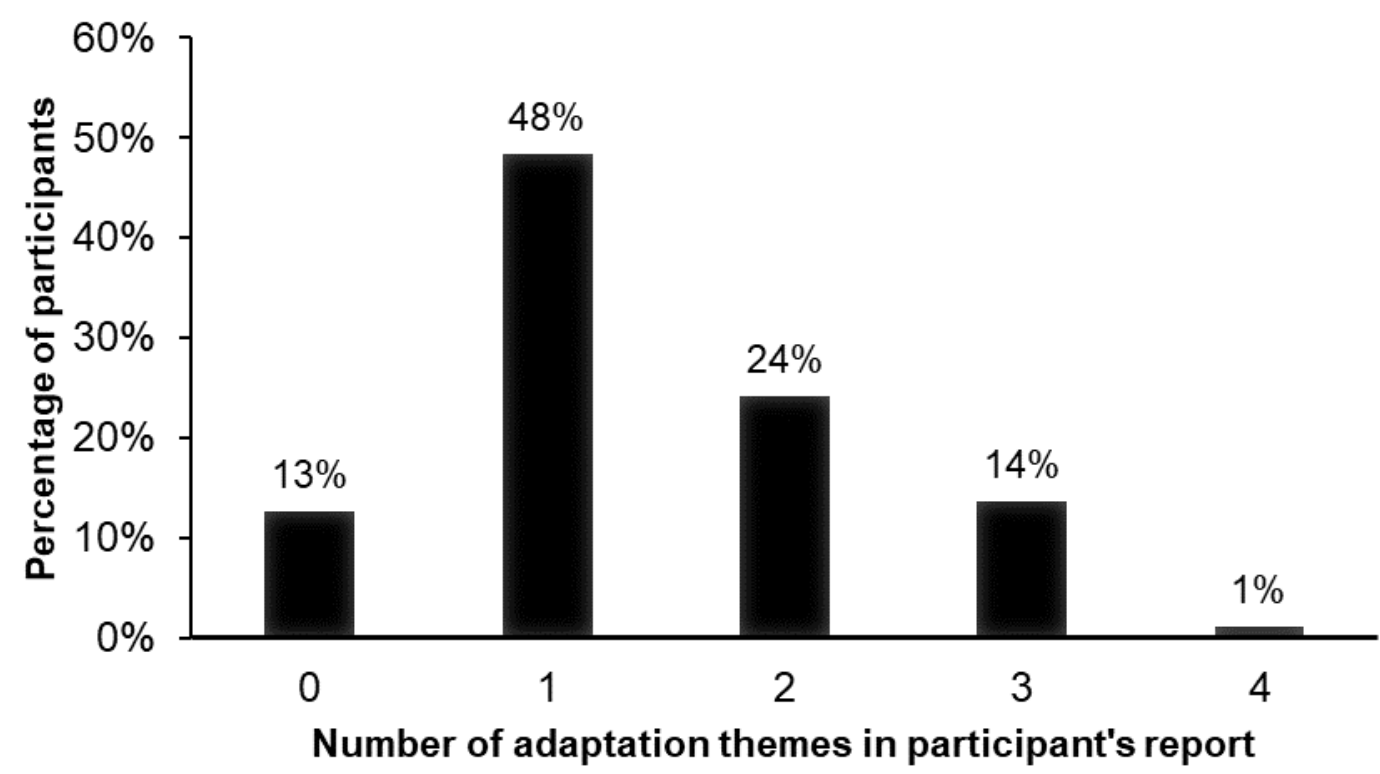

